\title{
Morphology and Selected Properties of Core/Shell ZnTe-Based Nanowire Structures Containing ZnO
}

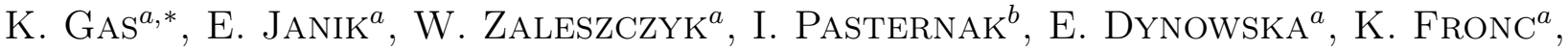 \\ V. Kolkovsky ${ }^{a}$, S. Kret ${ }^{a}$, J.F. Morhange ${ }^{c}$, A. Reszka $^{a}$, M. Wiater $^{a}, \mathrm{~W}$ Caliebe $^{d}$, \\ G. KARCzeWski ${ }^{a}$, B.J. KowAlski ${ }^{a}$, W. SzUszkiewiCz ${ }^{a}$ AND T. WojtowiCz ${ }^{a}$ \\ ${ }^{a}$ Institute of Physics, PAS, al. Lotników 32/46, 02-668 Warszawa, Poland \\ ${ }^{b}$ Institute of Electron Technology, al. Lotników 32/46, 02-668 Warsaw, Poland \\ ${ }^{c}$ Institut des Nanosciences de Paris, UMR 7588, UMPC, 140 rue de Lourmel, 75015 Paris, France \\ ${ }^{d}$ Hamburger Synchrotronstrahlungslabor HASYLAB am Deutschen Elektronen-Synchrotron \\ DESY, Notkestr. 85, D-22603 Hamburg, Germany
}

\begin{abstract}
We report on an approach to fabricate ZnTe-based core/shell radial heterostructures containing $\mathrm{ZnO}$, as well as on some of their physical properties. The molecular beam epitaxy grown ZnTe nanowires constituted the core of the investigated structures and the ZnO shells were obtained by thermal oxidation of ZnTe NWs. The influence of the parameters characterizing the oxidation process on selected properties of core/shell NWs were examined. Scanning electron microscopy revealed changes of the NWs morphology for various conditions of the oxidation process. X-ray diffraction, high resolution transmission electron microscopy, and Raman scattering measurements were applied to reveal the presence of ZnTe single crystal core and polycrystalline ZnO-shell of investigated structure.
\end{abstract}

PACS: 68.37.Hk, 68.37.Lp, 68.65.La, 81.07.Vb

\section{Introduction}

Quasi one-dimensional (1D) semiconductor nanostructures like, e.g., nanowires (NWs) have recently attracted a great deal of attention due to their interesting physical properties which make them potential candidates as "building blocks" in electronic and photonic nanodevices [1]. Nanostructures containing $\mathrm{ZnO}$ are of particular interest for these applications because of its wide direct band-gap $(3.37 \mathrm{eV}$ at $295 \mathrm{~K})$ and a large exciton binding energy $(60 \mathrm{meV})$. The non intentionally doped $\mathrm{ZnO}$ usually exhibits $n$-type conductivity and it is difficult to convert this material to $p$-type semiconductor. It is the reason that the practical applications of $\mathrm{ZnO}$ as a basic material in many devices is rather limited. On the other hand a combination of $\mathrm{ZnO}$ and $\mathrm{ZnTe}$, another direct wide-bandgap (2.26 eV at room temperature) semiconductor, has proved successful as, e.g., efficient solar cells [2]. Contrary to $\mathrm{ZnO}, \mathrm{ZnTe}$ can be obtained as a highly $p$-type doped material. Moreover, it was already shown that thermal oxidation of nitrogen-doped ZnTe allows to obtain p-type $\mathrm{ZnO}$ [3]. Up till now the fabrication of $\mathrm{ZnTe} / \mathrm{ZnO}$ core/shell NW structures was realized by a

\footnotetext{
* corresponding author; e-mail: kgas@ifpan.edu.pl
}

combination of two different growth methods. Molecular beam epitaxy (MBE) for the ZnTe core and atomic layer leposition (ALD) for $\mathrm{ZnO}$ shell $[4,5]$. In this paper we propose and report on a quite different technique, which instead of the overgrowth, employs the partial oxidation of ZnTe NWs into ZnTe/ZnO core/shell structures. We show that this can be an alternative method to fabricate this kind of heterostructures. Furthermore, such a process decreases the cores' diameters, thus increasing a chance to observe size effects in the NW structures.

\section{Experimental details}

ZnTe-based core/shell NW heterostructures were obtained using the thermal oxidation of $\mathrm{ZnTe} \mathrm{NWs}$ grown on GaAs or Si substrates by MBE employing a goldcatalyzed vapor-liquid-solid (VLS) mechanism [6-8]. Both "as-grown" ZnTe NWs on their original substrates, as well as samples containing these NWs mechanically removed from original substrate and deposited onto $\mathrm{Si}$ wafers were oxidized. Oxidation was performed by annealing in the presence of a flowing $\mathrm{O}_{2}$ gas (in the rapid thermal processing system - RTP AST SHS-100) or in a furnace under ambient atmosphere. Times and temperatures of oxidation process were in the range 3 min $-5 \mathrm{~h}$ and $150-600^{\circ} \mathrm{C}$, respectively. The surface morphology of 
the nanostructures was examined by Zeiss Auriga scanning electron microscopy (SEM). Structural characterization of the samples was carried out by X-ray diffraction (XRD) and high resolution transmission electron microscopy (HR TEM). The XRD patterns were measured at the W1 beamline at DESY-Hasylab using monochromatic synchrotron radiation $(\lambda=1.54056 \AA)$. The symmetrical $\omega-2 \theta$ scan (allowing to detect the lattice planes of NWs parallel to the crystallographic orientation of the substrate) were applied. HR TEM investigation was performed with a JEOL 2000EX microscope operating at $200 \mathrm{kV}$. The room temperature Raman scattering measurements were performed using a Jobin-Yvon U1000 double monochromator with a spectral resolution close to $1 \mathrm{~cm}^{-1}$. Excitation in near resonance conditions was achieved using the $514.5 \mathrm{~nm}$ line of the Argon laser.

\section{Results}

In order to optimize oxidation process the ZnTe NWs were oxidized under different conditions (temperature, time, atmosphere). After the oxidation process, the NWs' surface morphology is changed and their diameters have slightly increased as it is shown in Fig. 1. NWs conserve their shape for the temperatures of annealing below

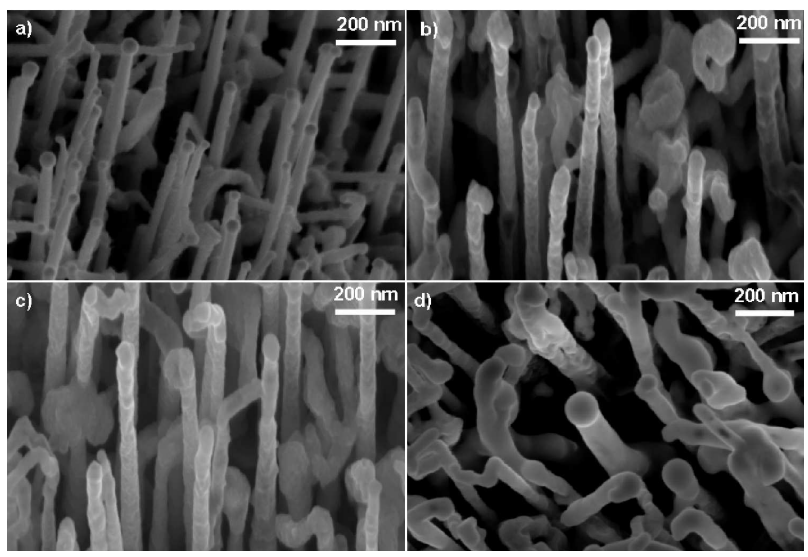

Fig. 1. SEM images of the ZnTe NWs grown on (110)-oriented GaAs, oxidized on the original substrate in $\mathrm{O}_{2}$ gas flow during $30 \mathrm{~min}$ at different temperatures (a) as grown sample, (b) after annealing at $360{ }^{\circ} \mathrm{C}$, (c) $400{ }^{\circ} \mathrm{C}$, and (d) $460{ }^{\circ} \mathrm{C}$.

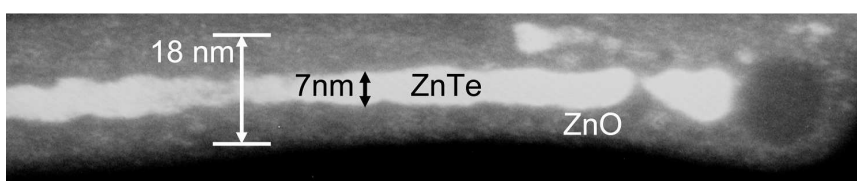

Fig. 2. Dark field TEM image of oxidized ZnTe NW grown on (111)-oriented $\mathrm{Si}$ substrate (oxidation process of as-grown ZnTe NWs was performed at $395^{\circ} \mathrm{C}$ during $30 \mathrm{~min}$ in air).

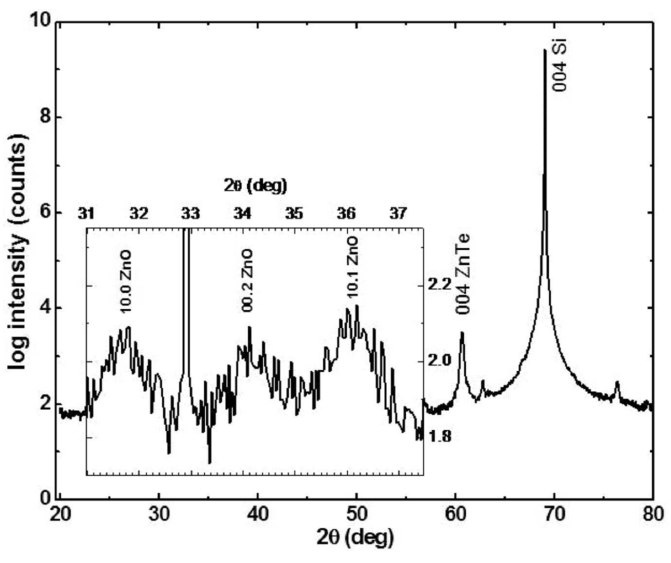

Fig. 3. Symmetrical X-ray diffraction pattern obtained for oxidized ZnTe NWs grown on (100)-oriented $\mathrm{Si}$ substrate (oxidation process of as-grown $\mathrm{ZnTe} \mathrm{NWs}$ was performed at $395^{\circ} \mathrm{C}$ during $30 \mathrm{~min}$ in air).

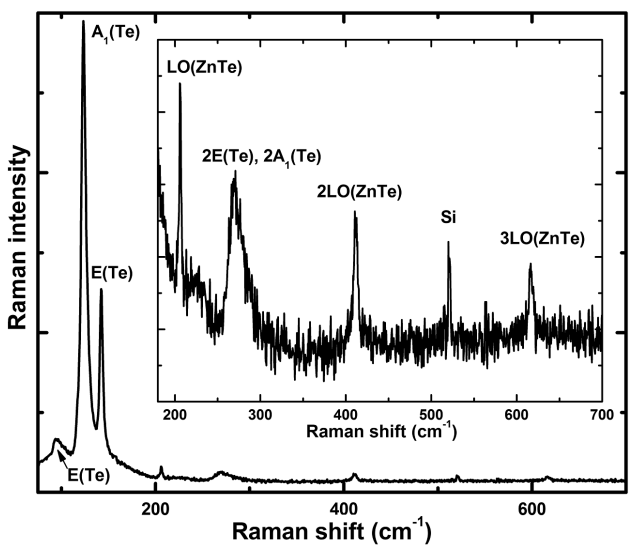

Fig. 4. Raman spectrum taken on oxidized ZnTe NWs grown on (100)-oriented Si substrate (oxidation process of as-grown ZnTe NWs was performed at $395^{\circ} \mathrm{C}$ during 30 minutes in air).

$500{ }^{\circ} \mathrm{C}$. The TEM investigations confirmed that that it is possible to obtain core/shell radial heterostructures by a proper selection of the oxidation process conditions. For the ZnTe NWs grown on the (111)-oriented Si substrate and oxidized in air at $395^{\circ} \mathrm{C}$ during $30 \mathrm{~min}$, the dark field TEM image (Fig. 2) obtained from 111 reflected beam of ZnTe shows that only a part of the NW has been transformed into polycrystalline $\mathrm{ZnO}$ while the rest of the NW remains unchanged. In this case, the diameter of the monocrystalline $\mathrm{ZnTe}$ core is about $7 \mathrm{~nm}$ and the thickness of the shell is about $2 \mathrm{~nm}$. XRD symmetrical $\omega-2 \theta$ pattern obtained for the ZnTe NWs grown on the (100)-oriented Si substrate oxidized in air at $395^{\circ} \mathrm{C}$ during $30 \mathrm{~min}$ (Fig. 3) indicate a monocrystalline nature of the ZnTe core, which has the same crystallographic orientation as that of the (100)-oriented Si substrate (reflections of ZnTe NWs indexed as 004 visible in the diffraction pattern taken at the symmetrical measurement mode 
corresponding to the analogous reflections of the Si substrate). Apart from the zinc blende ZnTe the XRD pattern also shows the presence of polycrystalline wurtzite $\mathrm{ZnO}$ (reflections of $\mathrm{ZnO}$ indexed as 10.0, 00.2, 10.1). The Fig. 4 gives an example of the Raman scattering spectrum taken on the same sample. The lines at the frequencies of $206,411.5$, and $617 \mathrm{~cm}^{-1}$ well correspond to the optical phonon mode (LO) of bulk ZnTe and its overtones, respectively. The large peaks centered at about $92 \mathrm{~cm}^{-1}$, $123 \mathrm{~cm}^{-1}$, and $142 \mathrm{~cm}^{-1}$ coincide with the phonon modes of trigonal Te with $\mathrm{E}, \mathrm{A}_{1}$, and E symmetry, respectively [9]. The structure near $270 \mathrm{~cm}^{-1}$ probably can be assigned to the overtone of the phonon lines at $123 \mathrm{~cm}^{-1}$, and $142 \mathrm{~cm}^{-1}$. Tellurium precipitates usually exist in MBE grown ZnTe and this material presents high scattering cross section. The line centered at $520 \mathrm{~cm}^{-1}$ is due to the scattering on the optical phonon mode of Si. No Raman scattering can be attributed to the $\mathrm{ZnO}$ shell.

\section{Conclusions}

The ZnTe-based and ZnO coated core/shell NWs were successfully obtained by thermal oxidation of ZnTe NWs. The SEM investigation revealed the change in the NWs' morphology after oxidation process. TEM studies shown that oxidized NWs consist of two different materials $\mathrm{ZnTe}$ core and $\mathrm{ZnO}$ shell. The presence of zinc blende ZnTe core in the heterostructures was confirmed by both Raman scattering and XRD measurement. Apart from the ZnTe core the XRD studies revealed the presence of polycrystalline wurtzite $\mathrm{ZnO}$ in the oxidized samples.

\section{Acknowledgments}

The research was partially supported by the European Union within European Regional Development Found, through grant Innovate Economy (POIG.01.01.02-00008/08), and by the EC-RIA under FP6 "Structuring the
European Research Area" Programme through the Integrated Infrastructure Initiative "Integrating Activity on Synchrotron and Free Electron Laser Science", Contract RII3-CT-2004-506008.

\section{References}

[1] Y. Xia, P. Yang, Y. Sun, Y. Wu, B. Mayers, B. Gates, Y. Yin, F. Kim, H. Yan, Adv. Mater. 15, 353 (2003).

[2] H.Y. Chao, J.H. Cheng, J.Y. Lu, Y.H. Chang, C.L. Cheng, Y.F. Chen, Supperlatt. Microstruct. 47, 160 (2010).

[3] E. Przeździecka, E. Kamińska, K.P. Korona, E. Dynowska, W. Dobrowolski, R. Jakieła, Ł. Kłopotowski, J. Kossut, Semicond. Sci. Technol. 22, 10 (2007).

[4] E. Janik, A. Wachnicka, E. Guziewicz, M. Godlewski, S. Kret, W. Zaleszczyk, E. Dynowska, A. Presz, G. Karczewski, T. Wojtowicz, Nanotechnology 21, 015302 (2010).

[5] A. Szczepanik, Ł. Wachnicki, M. Godlewski, E. Guziewicz, K. Kopalko, E. Janik, E. Łusakowska, A. Czerwiński, M. Płuska, S.A. Yatsunenko, J. Phys. Conf. Series 146, 012017 (2009).

[6] R.S. Wagner, W.C. Ellis, Appl. Phys. Lett. 4, 89 (1964).

[7] E. Janik, J. Sadowski, P. Dłużewski, S. Kret, L.T. Baczewski, A. Petroutchik, E. Łusakowska, J. Wróbel, W. Zaleszczyk, G. Karczewski, T. Wojtowicz, A. Presz, Appl. Phys. Lett. 89, 133114 (2006).

[8] E. Janik, P. Dłużewski, S. Kret, A. Presz, H. Kirmse, W. Neumann, W. Zaleszczyk, L.T. Baczewski, A. Petroutchik, E. Dynowska, J. Sadowski, W. Caliebe, G. Karczewski, T. Wojtowicz, Nanotechnology 18, 475606 (2007).

[9] A.S. Pine, G. Dresselhaus, Phys. Rev B 4, 356 (1971). 